\title{
Efficacy and Safety of Traditional Chinese Medicine in Idiopathic Pulmonary Fibrosis: A Meta-Analysis
}

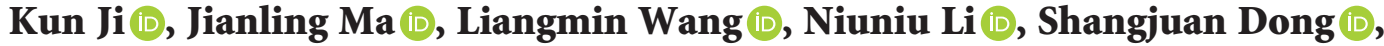 \\ and Liqing Shi \\ Department of Respiratory Medicine, Dongfang Hospital, Beijing University of Traditional Chinese Medicine, \\ Beijing 100078, China \\ Correspondence should be addressed to Liqing Shi; shiliqing3662@sina.com
}

Received 23 August 2019; Revised 3 December 2019; Accepted 20 December 2019; Published 13 February 2020

Academic Editor: Christopher Worsnop

Copyright (c) 2020 Kun Ji et al. This is an open access article distributed under the Creative Commons Attribution License, which permits unrestricted use, distribution, and reproduction in any medium, provided the original work is properly cited.

Objective. To evaluate the efficacy and safety of traditional Chinese medicine (TCM) on lung function and quality of life of idiopathic pulmonary fibrosis (IPF) patients by meta-analysis. Methods. Randomized controlled trials (RCTs) related to TCM and IPF were searched on PubMed, EMBASE Cochrane Library, ClinicalTrials, China National Knowledge Infrastructure (CNKI), Wanfang Database, Chin VIP Information (VIP), and Chinese Biomedical Database (CBM) until December 2018. Standard mean difference (SMD) and $95 \% \mathrm{CI}$ were calculated for the measurements related to lung function (FEV1/FVC, FVC\%, FEV1\%, TLC\%, DLCO\% or DLCO, and $\mathrm{VC} \%$ ) and other parameters ( $\mathrm{PO}_{2}, 6 \mathrm{MWD}$, and SGRQ) when comparing TCM treatment to the control group. Relative risk (RR) and 95\% CI of adverse events (AEs) were calculated to assess the safety of TCM. Results. A total of 40 RCTs comparing TCM to western medicine (WM) and involving 3194 IPF patients were eligible for the meta-analysis. The pooled results showed that TCM treatment improved significantly $\mathrm{PO}_{2}(\mathrm{SMD}=0.80,95 \% \mathrm{CI} 0.54$ to $1.06, p<0.001)$, FEV $1 \%$ (SMD $=0.57,95 \% \mathrm{CI} 0.42$ to $0.71, p<0.001$ ), $\mathrm{DLCO} \%$ $(\mathrm{SMD}=0.38,95 \%$ CI 0.28 to $0.48, p<0.001), 6 \mathrm{MWD}$ (SMD $=0.70,95 \%$ CI 0.56 to $0.84, p<0.001)$ and other measurements and reduced SGRQ scores $(\mathrm{SMD}=-0.51,95 \% \mathrm{CI}-0.70$ to $-0.22, p<0.001)$. Subgroup analysis of different study durations $(3$ months, $\geq 6$ months) and comparison models (TCM vs. WM, TCM + WM vs. WM or TCM vs. placebo) showed similar results. No significant difference of risk of AEs was observed between both groups $(\mathrm{RR}=0.66,95 \% \mathrm{CI}: 0.27-1.60, p=0.352)$. There was no obvious publication bias, and the pooled results were stable according to sensitivity analysis. Conclusion. To the best of our knowledge, the present study had the largest sample size. Our results indicated that TCM treatment may help provide benefit to the lung function, exercise capacity, and quality of life of IPF patients, alone or combined with WM, when compared to WM. More rigorous RCTs were needed in the future.

\section{Introduction}

Idiopathic pulmonary fibrosis (IPF) is a rare disease characterized by chronic, progressive, and fibrosing interstitial pneumonia with undetermined etiology. It is the most common type of idiopathic interstitial pneumonia. The incidence of IPF varies in different populations and has risen over time. It is estimated to range from 2.8 to 18 cases in the western countries and from 0.5 to 4.2 cases in the Asia and South American, per 100000 people per year [1,2]. Although the exact etiology remains unclear, multiple environmental exposures and genetic factors have been implicated [3].

IPF is a life-threatening disease with extremely poor prognosis. As the disease progresses, the lung function continues to decline despite treatments, which ultimately leads to respiratory failure and even death. The median survival time from diagnosis is only 2 to 3 years [4]. Currently, the pharmacological therapy for IPF includes glucocorticoid, immunosuppressive or cytotoxic agents, tyrosine kinase inhibitor, and antifibrotic agents. Yet, randomized trials have identified some drugs were potentially ineffective or harmful $[5,6]$, whereas only two, pirfenidone and nintedanib, were proved to be effective disease-modifying therapies for IPF [7, 8]. Besides pharmacological therapy, lung transplantation can prolong survival with a 5year survival of near $50 \%$ and improve quality of life of IPF patients [9]. However, only a few patients can receive transplantation due to the high expense and lack of donors 
organs. Overall, there is an urgent need to develop new therapy for IPF with clear effect and less adverse events.

In recent years, traditional Chinese medicine (TCM) has been reported to have effect on IPF in animal models and patients [10]. TCM was recommended as an experimental therapy for IPF, and many kinds of TCM were widely clinically used in China. However, due to the lack of largescale, multicentered, randomized controlled trials, the treatment efficacy of TCM on IPF is still in controversy. In the present study, we aimed to evaluate the efficacy of TCM on IPF by meta-analysis.

\section{Methods}

2.1. Literature Search. Literature search was performed on English databases including PubMed, EMBASE, Cochrane Library, and ClinicalTrials, and Chinese databases including China National Knowledge Infrastructure (CNKI), Wanfang Database, Chin VIP Information (VIP) and Chinese Biomedical Database (CBM) until December 2018. Different combinations of the following terms were used: (IPF OR pulmonary fibrosis OR lung fibrosis) AND (Chinese medicine OR herbal medicine OR herbal drug).

2.2. Including and Excluding Criteria. Eligible studies should fulfill the following criteria: (1) randomized controlled trials (RCTs) with regards to the efficacy of Tradition Chinese Medicine (TCM) to idiopathic pulmonary fibrosis; (2) comparing TCM vs. placebo or TCM vs. western med (WM) or TCM + WM vs. WM; (3) reporting at least one of the following outcomes with measured values or the percentages to the predicted value: VC (vital capacity), FEV1 (forced expiratory volume in one second), FVC (forced vital capacity), TLC (total lung capacity), FEV1/FVC, DLCO (diffusion capacity of the lung for carbon monoxide), $\mathrm{PO}_{2}$ (partial pressure of oxygen), 6MWD (6-minute walking distance), SGRQ scores (St. George's respiratory questionnaire), CAT (COPD assessment test), SF-36 Health Survey questionnaire, ATAQ-IPF (a tool to assess quality of life in idiopathic pulmonary fibrosis), and AEs; (4) lasting at least of 3 months or 12 weeks. Cases reports, reviews, non-RCTs, duplications, and those comparing different TCMs or assessing nondrug treatments (acupuncture and taichi for example) were all discarded.

2.3. Quality Assessment. The quality of all eligible studies was assessed according to the Jadad scale comprised of three items: randomization, blinding, withdrawals, and dropouts [11]. Zero, one, or two scores were given according to the description and appropriateness of these items. A study with a total score $\geq 3$ was considered to be of high quality; otherwise, it was of low quality.

2.4. Data Extraction. The following information of each eligible study were extracted: the first author, year of publishing, regimens of the treatment group and control group, number and mean age of patients in each group, duration of the trial and the measurements of clinical outcomes. Specifically, the literature search, study filtering, quality assessment, and data extraction were performed by two independent researchers. If disagreements occurred in any step, the researchers would discuss the details until they reached a consensus.

2.5. Statistics. The heterogeneity between studies was calculated by $I^{2}$ statistics. If there was low heterogeneity $\left(I^{2}<50 \%\right)$, the fixed-effect model would be used. Otherwise, the random-effect model was used. Standard mean difference (SMD) and the 95\% CI of each outcome measurement were calculated to assess the difference of treatment efficacy between different regimens. Relative risk (RR) and 95\% CI of AEs were calculated to assess the safety of TCM. Additionally, we performed the subgroup analysis according to the trial duration and comparison of regimens as follows:

(1) Duration subgroups: 3 months (or 12 weeks); 6 months (or 24 weeks) or more

(2) Comparison subgroups: TCM vs. WM; TCM + WM vs. WM or TCM vs. placebo

However, only the subgroups involving at least 3 studies were analyzed. Sensitivity analysis was performed to identify if any study had significant impact on the pooled results of meta-analysis. Egger's test and funnel plot were used to assess the publication bias. All statistics were performed by using STATA 11.0 (StataCorp LP, TX, USA). $p$ value less than 0.05 indicated statistical significance.

\section{Results}

3.1. Characteristics of All Eligible Studies. Literature search retrieved a total of 1477 published articles, of which 1403 did not fulfill the inclusion/exclusion criteria and were discarded after screening the titles and abstracts. The remaining articles were reviewed for the full text and 34 articles were subsequently excluded according to the inclusion/exclusion criteria. Finally, 40 studies were included in the present metaanalysis [12-51]. The flowchart of literature review is shown in Figure 1.

A total of 3194 participants were involved in the analysis, of which 1647 were in the intervention group (TCM only or $\mathrm{TCM}+\mathrm{WM}$ ) and 1547 were in the control group (WM or placebo). The sample size of each study ranged from 34 to 324 and the duration varied from 3 months (or 12 weeks) to 18 months. As for the regimen, 29 studies compared the effect of TCM plus WM to WM alone in IPF, of which 21 were TCM + glucocorticoid versus glucocorticoid (prednisone or dexamethasone) $[12-14,17,18,20,21$, $23,24,29,31,32,35-37,39,40,42,44,48,49]$, 6 were $\mathrm{TCM}+\mathrm{N}$-acetylcysteine versus $\mathrm{N}$-acetylcysteine [26, 28, 33-35, 40], one was TCM + edaravone versus edaravone [30] and one was TCM + pirfenodone versus pirfenodone [37]. The other 11 studies compared TCM versus placebo [43] or TCM versus WM $[14,16,19,22,25,27,28,41,46,51]$. Additionally, one trial [22] assigned patients to different dosage groups (high or low dosage group), and therefore, 


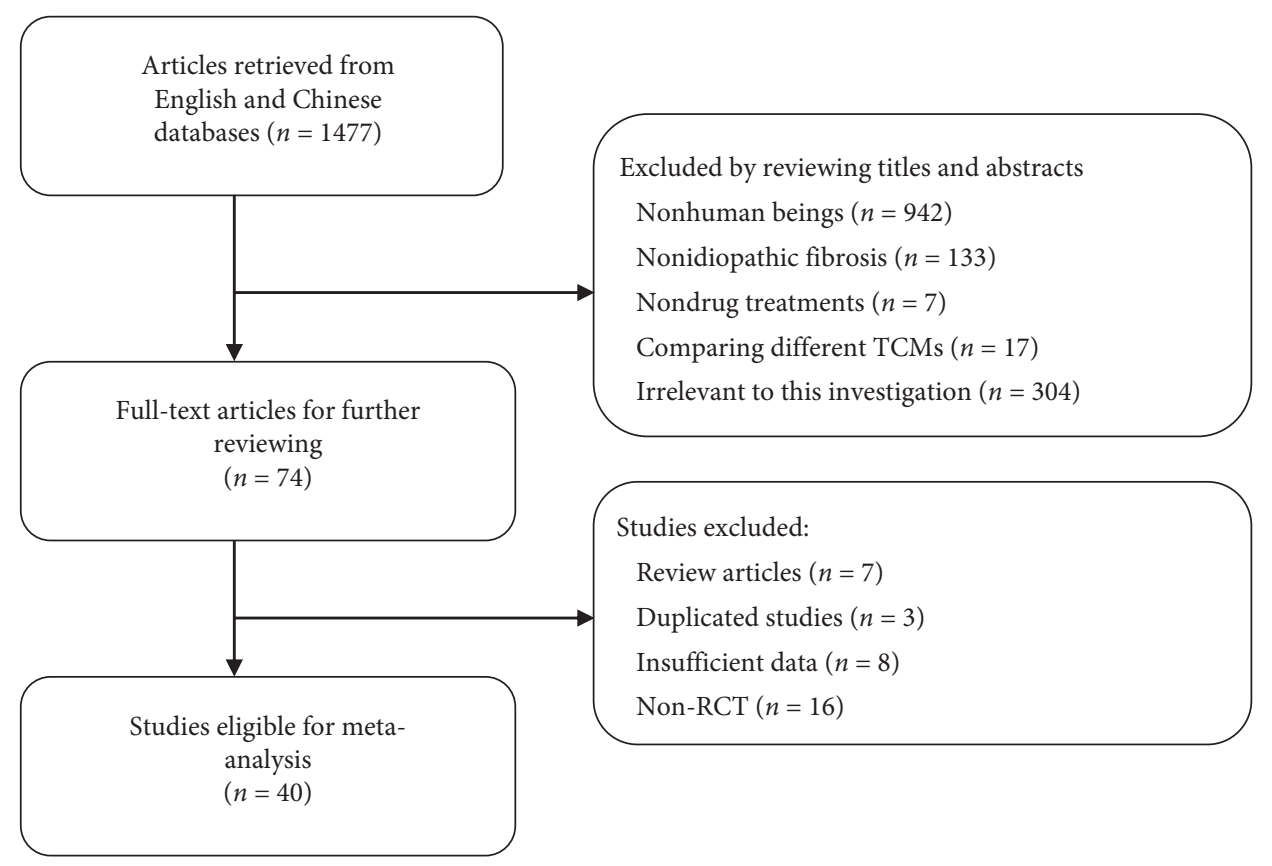

FIGURE 1: Flowchart of literature reviewing and filtering.

each dosage group was separately pooled in the metaanalysis. None of the included studies reported results of CAT, SF-36, and ATAQ-IPF, and these outcomes were not analyzed in the present study. We also assessed the quality of each randomized trial by Jadad scores and found that 5 studies were of high quality (Jadad scores $\geq 3$ ) $[22,23,43,48,51]$, whereas the rest were of low quality. The characteristic of all included studies are listed in Table 1.

3.2. Lung Function. There were 6 and 11 studies evaluating the change of FEV1/FVC or FVC\% in both groups, respectively (Table 2). High heterogeneities were found in both analyses $\left(I^{2}>50 \%\right)$ and random-effect model was applied. The pooled results showed significant differences of FEV1/ $\mathrm{FVC}(\mathrm{SMD}=0.90 .95 \% \mathrm{CI} 0.48$ to $1.31, p<0.001)$ and $\mathrm{FVC} \%$ $(\mathrm{SMD}=0.60,95 \%$ CI 0.40 to $0.80, p<0.001)$ between TCM and control groups.

We included 10 studies measuring FEV1\% in IPF patients in the meta-analysis with a total of 781 cases (Table 2). There was low heterogeneity between studies $\left(I^{2}=14.1 \%\right)$ so that the fixed-effect model was used. The pooled analysis showed a significant improvement of FEV1\% in the TCM group than that in the control group $(\mathrm{SMD}=0.57,95 \% \mathrm{CI}$ 0.42 to $0.71, p<0.001$ ) as shown in Figure 2.

Additionally, 20 studies comprising 1518 patients reported changes of DLCO\% before and after intervention in both groups (Table 2), and there was a moderate heterogeneity $\left(I^{2}=39.4 \%\right)$. Pooled analysis (Figure 3 ) in the fixedeffect model showed TCM treatment significantly improved $\mathrm{DCLO} \%$ when comparing to the control group $(\mathrm{SMD}=0.38$, 95\% CI 0.28 to $0.48, p<0.001)$.

We also compared the other measurements related to lung function (FVC\%, TLC\%, DLCO, VC\%) between TCM and control groups by meta-analysis (Table 2). There was no or low heterogeneity, and pooled results indicated significant improvements of these measurements in the TCM group than that in the control group ( $p$ for SMD $<0.001$ ).

3.3. Other Parameters. A total of 18 studies assessed the effect of TCM on $\mathrm{PO}_{2}$ change in IPF with 717 patients in the treatment group and 705 in control group (Table 2). There was a high heterogeneity $\left(I^{2}=81.7 \%\right)$ so the random-effect model was used. After pooling analysis, the SMD was 0.80 (95\% CI 0.54 to $1.06, p<0.001$ ), indicating that the TCM group had significant improvement of $\mathrm{PO}_{2}$ compared to the control group (Figure 4).

There were 11 studies with 828 IPF patients measuring $6 \mathrm{MWD}$ and were included in our analysis (Table 2). Low heterogeneity was found $\left(I^{2}=23.3 \%\right)$ and then the fixedeffect model was applied. The pooled analysis indicated a significant improvement of 6MWD in TCM group compared with the control group $(\mathrm{SMD}=0.70,95 \%$ CI 0.56 to $0.84, p<0.001)$ as shown in Figure 5.

SGRQ scores were reported in 10 studies involving 342 patients in the TCM group and 336 patients in the control group (Table 2). The random-effect model was used due to obvious heterogeneity $\left(I^{2}=69.8 \%\right)$. After pooling analysis (Figure 6), TCM treatment significantly decreased SGRQ scores compared with the control group $(\mathrm{SMD}=-0.51,95 \%$ CI -0.70 to $-0.22, p<0.001)$.

3.4. Subgroup Analysis. Subgroup analyses were performed according to study duration, so as to assess the short term (3 months) and long term (6 months or more) effect, and different comparison models (TCM vs. WM, TCM + WM vs. WM or TCM vs. placebo). We only analyzed those subgroups involving at least 3 studies. For the duration subgroups (Table 3), TCM treatment had significant 
TABLE 1: Characteristics of all studies included in the meta-analysis.

\begin{tabular}{|c|c|c|c|c|c|c|c|}
\hline \multirow{2}{*}{ Study } & \multicolumn{2}{|l|}{ Regimen } & \multirow{2}{*}{$\begin{array}{c}\text { No. of } \\
\text { patients* }\end{array}$} & \multirow{2}{*}{ Age (years) ${ }^{*}$} & \multirow{2}{*}{ Duration $^{\#}$} & \multirow{2}{*}{ Outcomes } & \multirow{2}{*}{ Jadad } \\
\hline & Treatment & Control & & & & & \\
\hline Cao et al. [12] & $\begin{array}{l}\text { Tongluo Huaxian } \\
\text { granules + PDN }\end{array}$ & PDN & $30 / 30$ & $60.27 / 61.54$ & $6 \mathrm{M}$ & VC\%, TLC\% & 2 \\
\hline Dong [13] & $\begin{array}{l}\text { Kangxian Shufei } \\
\text { granules + PDN }\end{array}$ & $\mathrm{PDN}$ & $33 / 33$ & $59.11 / 57.7$ & $3 \mathrm{M}$ & $\begin{array}{c}\mathrm{DLCO} \%, \mathrm{PO}_{2}, \mathrm{FEV} 1 \%, \mathrm{FVC} \\
\%\end{array}$ & 1 \\
\hline Fan et al. [14] & Kechuanting granules & PDN & $32 / 31$ & $54.11 / 53.03$ & $6 \mathrm{M}$ & FEV1\%, FEV1/FVC, & 1 \\
\hline Tan and Li [15] & $\begin{array}{l}\text { Shuizhi Tongluo } \\
\text { capsule + PDN }\end{array}$ & PDN & $31 / 31$ & $61.1 / 63.67$ & $3 \mathrm{M}$ & $\mathrm{DLCO} \%, \mathrm{PO}_{2}$ & 1 \\
\hline Wang et al. [16] & Yangyin Yiqi Misture & $\mathrm{PDN}$ & $34 / 30$ & $66.07 / 63.13$ & $6 \mathrm{M}$ & DLCO\%, FEV1\%, FVC\%, & 2 \\
\hline Weng and Ma [17] & Qingjin decoction + PDN & PDN & $42 / 42$ & $52.66 / 53.33$ & $6 \mathrm{M}$ & $\mathrm{PO}_{2}, \mathrm{FEV} 1 \%$ & 2 \\
\hline Chen et al. [18] & $\begin{array}{l}\text { Huaxian Pogu } \\
\text { formula + PDN }\end{array}$ & PDN & $25 / 25$ & $63 / 65$ & $3 \mathrm{M}$ & VC\%, DLCO $\%$, TLC\%, $\mathrm{PO}_{2}$ & 1 \\
\hline Fan et al. [19] & Feixiantong decoction & NAC & $22 / 21$ & $60.98 / 65.12$ & $12 \mathrm{~W}$ & VC\%, DLCO\%, TLC\% & 2 \\
\hline Feng et al. [20] & $\begin{array}{c}\text { Feixiankang } \\
\text { granules + PDN }\end{array}$ & PDN & $30 / 30$ & - & $6 \mathrm{M}$ & $\mathrm{DLCO} \%, \mathrm{PO}_{2}$ & 1 \\
\hline Gan et al. [21] & Huaxian decoction + PDN & PDN & $26 / 27$ & $63.2 / 64.5$ & $3 \mathrm{M}$ & DLCO & 2 \\
\hline Fan et al. [22] & $\begin{array}{c}\text { Feitong oral liquid (high } \\
\text { dose) }\end{array}$ & PDN & $73 / 65$ & $59.01 / 58.27$ & $3 \mathrm{M}$ & $\begin{array}{c}\mathrm{DLCO} \%, \mathrm{TLC} \%, \mathrm{PO}_{2}, \\
6 \mathrm{MWD}\end{array}$ & 5 \\
\hline Fan et al. [22] & $\begin{array}{l}\text { Feitong oral liquid (low } \\
\text { dose) }\end{array}$ & PDN & $66 / 65$ & $57.38 / 58.27$ & $3 \mathrm{M}$ & $\begin{array}{c}\mathrm{DLCO} \%, \mathrm{TLC} \%, \mathrm{PO}_{2}, \\
6 \mathrm{MWD}\end{array}$ & 5 \\
\hline Xu et al. [23] & $\begin{array}{l}\text { Bufei Yishen Huoxue } \\
\text { decoction + PDN }\end{array}$ & PDN & $35 / 35$ & $52.32 / 51.51$ & $12 \mathrm{~W}$ & $\mathrm{DLCO} \%, \mathrm{PO}_{2}, \mathrm{FVC} \%$, & 3 \\
\hline Chen [24] & Danhong injection + PDN & PDN & $45 / 45$ & $50.2 / 49.5$ & $12 \mathrm{~W}$ & DLCO & 2 \\
\hline Li et al. [25] & Feibitongfang & PDN & $23 / 22$ & $59.97 / 64.59$ & $12 \mathrm{~W}$ & $\mathrm{VC} \%, \mathrm{TLC} \%, \mathrm{PO}_{2}$ & 2 \\
\hline Yan et al. [26] & $\begin{array}{l}\text { Buyang Huanwu } \\
\text { decoction + NAC }\end{array}$ & NAC & $60 / 60$ & $63.5 / 63.4$ & $6 \mathrm{M}$ & DLCO $\%, \mathrm{PO}_{2}, \mathrm{FEV} 1 \%$ & 2 \\
\hline Li et al. [27] & Yangyin Yifei Tongluo Wan & NAC & $34 / 30$ & $58.23 / 59.98$ & $3 \mathrm{M}$ & VC\%, DLCO\%, TLC\% & 2 \\
\hline Liu et al. [28] & $\begin{array}{c}\text { Xuefu Zhuyu } \\
\text { capsule + NAC }\end{array}$ & NAC & $18 / 16$ & - & $18 \mathrm{M}$ & $\begin{array}{c}\mathrm{DLCO} \%, \mathrm{TLC} \%, \mathrm{PO}_{2}, \mathrm{FVC} \\
\%, 6 \mathrm{MWD}\end{array}$ & 2 \\
\hline Song [29] & $\begin{array}{c}\text { Xuefu Zhuyu } \\
\text { decoction + PDN }\end{array}$ & PDN & $48 / 48$ & $62.1 / 63.27$ & $3 \mathrm{M}$ & FEV1\%, FVC\%, SGRQ & 2 \\
\hline Sun et al. [30] & Danhong injection + ED & $\mathrm{ED}$ & $35 / 35$ & $48.2 / 50.8$ & $12 \mathrm{~W}$ & DLCO, $\mathrm{PO}_{2}$ & 2 \\
\hline Yan [31] & Danhong injection + PDN & PDN & $34 / 34$ & $52.3 / 53.5$ & $12 \mathrm{~W}$ & DLCO & 1 \\
\hline Hu et al. [32] & $\begin{array}{l}\text { Yiqi Huayu Tongluo } \\
\text { decoction + PDN }\end{array}$ & PDN & $40 / 40$ & $53.87 / 54.19$ & $12 \mathrm{~W}$ & DLCO, $\mathrm{PO}_{2}$, SGRQ & 2 \\
\hline Wang et al. [33] & $\begin{array}{l}\text { Wenyang Huayu } \\
\text { decoction + NAC }\end{array}$ & NAC & $43 / 37$ & - & $3 \mathrm{M}$ & VC\%, DLCO\%, TLC\%, & 1 \\
\hline Zhao and $\mathrm{Wu}[34]$ & Danhong Injection + NAC & NAC & $40 / 40$ & $62.3 / 62.8$ & $12 \mathrm{~W}$ & DLCO, $\mathrm{PO}_{2}, \mathrm{FEV} 1 / \mathrm{FVC}$ & 1 \\
\hline Han et al. [35] & $\begin{array}{l}\text { Pingfeng Shengmai } \\
\text { powder + PDN }\end{array}$ & PDN & $47 / 47$ & $59.23 / 58.64$ & $3 \mathrm{M}$ & VC\%, DLCO $\%$, TLC\%, $\mathrm{PO}_{2}$ & 1 \\
\hline $\mathrm{Hu}[36]$ & $\begin{array}{l}\text { Pingfeng Shengmai } \\
\text { powder + PDN }\end{array}$ & PDN & $45 / 44$ & $56.21 / 56.68$ & $6 \mathrm{M}$ & FEV1\%, FVC\%, 6MWD & 1 \\
\hline Li et al. [37] & $\begin{array}{l}\text { Peiyuan Huoxue } \\
\text { decoction + PDN }\end{array}$ & PDN & $42 / 42$ & $59.4 / 59.8$ & $6 \mathrm{M}$ & FEV1/FVC & 1 \\
\hline Miao et al. [38] & Bushen Tongluo decoction & NAC & $28 / 27$ & $62.36 / 65.68$ & $12 \mathrm{~W}$ & $\begin{array}{l}\text { VC\%, DLCO } \%, 6 \mathrm{MWD}, \\
\text { SGRQ }\end{array}$ & 2 \\
\hline Wang [39] & $\begin{array}{c}\text { Xuefu Zhuyu } \\
\text { decoction + PDN }\end{array}$ & PDN & $162 / 162$ & $60.6 / 61.3$ & $24 \mathrm{~W}$ & $\mathrm{FVC} \%$ & 2 \\
\hline Xie and Tong [40] & $\begin{array}{c}\text { Yangyin Yiqi } \\
\text { Misture + DXM }\end{array}$ & DXM & $45 / 45$ & - & $3 \mathrm{M}$ & FEV1/FVC, 6MWD, SGRQ & 2 \\
\hline Xin and Feng [41] & Erjiaxiaozheng decoction & NAC & $25 / 24$ & $65.68 / 65.88$ & $6 \mathrm{M}$ & 6MWD, SGRQ & 1 \\
\hline Yuan et al. [42] & $\begin{array}{l}\text { Bufei Yishen Huoxue } \\
\text { Misture + DXM }\end{array}$ & DXM & $39 / 39$ & $68.56 / 69.61$ & $3 \mathrm{M}$ & $\begin{array}{l}\text { FEV1\%, FEV1/FVC, } \\
\text { 6MWD, SGRQ }\end{array}$ & 2 \\
\hline Cui et.al. [43] & $\begin{array}{c}\text { Fuzheng Tixie Souluo } \\
\text { decoction }\end{array}$ & Placebo & $30 / 31$ & $63.9 / 62.61$ & $12 \mathrm{~W}$ & $\begin{array}{l}\text { VC\%, DLCO } \% \text {, TLC\%, } \\
\text { FEV1\%, FVC\%, SGRQ }\end{array}$ & 4 \\
\hline $\begin{array}{l}\text { Deng and Wang } \\
{[44]}\end{array}$ & $\begin{array}{l}\text { Huangqi Taohong } \\
\text { decoction + PDN }\end{array}$ & PDN & $59 / 59$ & $64.06 / 63.21$ & $3 \mathrm{M}$ & $\mathrm{DLCO} \%, \mathrm{PO}_{2}, \mathrm{FVC} \%$ & 2 \\
\hline Feng and Sun [45] & Yifei Tongluo recipe + NAC & NAC & $31 / 31$ & $61 / 62.3$ & $6 \mathrm{M}$ & DLCO, 6MWD & 2 \\
\hline $\mathrm{Fu}$ and $\mathrm{Lu}[46]$ & Loubei Lengshu decoction & PDN & $50 / 50$ & - & $3 \mathrm{M}$ & DLCO\%, TLC\% & 2 \\
\hline
\end{tabular}


TABLE 1: Continued.

\begin{tabular}{|c|c|c|c|c|c|c|c|}
\hline \multirow{2}{*}{ Study } & \multicolumn{2}{|l|}{ Regimen } & \multirow{2}{*}{$\begin{array}{c}\text { No. of } \\
\text { patients* }\end{array}$} & \multirow{2}{*}{ Age (years)* } & \multirow{2}{*}{ Duration $^{\#}$} & \multirow{2}{*}{ Outcomes } & \multirow{2}{*}{ Jadad } \\
\hline & Treatment & Control & & & & & \\
\hline Gu [47] & $\begin{array}{c}\text { Qishufeixian } \\
\text { decoction + PFD }\end{array}$ & PFD & $30 / 30$ & $62.3 / 63.8$ & $6 \mathrm{M}$ & $\begin{array}{l}\text { FEV1\%, FEV1/FVC, FVC\%, } \\
\text { SGRQ }\end{array}$ & 2 \\
\hline Peng et al. [48] & Qigui recipe + PDN & PDN & $25 / 25$ & 58. & $12 \mathrm{~W}$ & 6MWD, SGRQ & 3 \\
\hline Wu [49] & Danhong injection $+\mathrm{PDN}$ & $\mathrm{PDN}$ & $30 / 30$ & $61.56 / 61.8$ & $12 \mathrm{~W}$ & $\mathrm{DLCO}, \mathrm{PO}_{2}$ & 2 \\
\hline $\mathrm{Xi}$ and Qin [50] & $\begin{array}{l}\text { Huaxian Tongluo } \\
\text { decoction + NAC }\end{array}$ & NAC & $30 / 30$ & $65.11 / 64.28$ & $3 \mathrm{M}$ & $\begin{array}{c}\mathrm{VC} \%, \text { DLCO } \%, \text { TLC } \%, \mathrm{PO}_{2} \\
\text { 6WMD, SGRQ }\end{array}$ & 2 \\
\hline Zhao et al. [51] & Fuzheng Huaxian formula & NAC & $30 / 24$ & $58 / 59$ & $3 \mathrm{M}$ & $\begin{array}{c}\text { DLCO } \%, \text { FVC\%, 6MWD, } \\
\text { SGRQ }\end{array}$ & 3 \\
\hline
\end{tabular}

${ }^{*}$ Treatment/control; ${ }^{*} \mathrm{M}=$ months, $\mathrm{W}$ = weeks; 6MWD: 6-minute walking distance; SGRQ: St. George's respiratory questionnaire; VC: vital capacity; FEV1: forced expiratory volume in one second; TLC: total lung capacity; FVC: forced vital capacity; DLCO: diffusion capacity of the lung for carbon monoxide; NAC: Nacetylcysteine; PDN: prednisone; DXM: dexamethasone; PFD: pirfenidone; ED: edaravone; \% indicates the percentage of measured value to predicted value.

TABLe 2: Meta-analysis of the efficacy of TCM on idiopathic pulmonary fibrosis.

\begin{tabular}{|c|c|c|c|c|c|c|}
\hline \multirow{2}{*}{ Measurements } & \multirow{2}{*}{ No. of studies } & \multirow{2}{*}{ No. of patients } & \multicolumn{3}{|c|}{ Effect size } & \multirow{2}{*}{$I^{2}(\%)$} \\
\hline & & & SMD & $95 \% \mathrm{CI}$ & $p$ & \\
\hline $\mathrm{PO}_{2}$ & 18 & $717 / 705$ & 0.80 & $0.54,1.06$ & $<0.001$ & 81.7 \\
\hline FEV1/FVC & 6 & $228 / 227$ & 0.90 & $0.48,1.31$ & $<0.001$ & 77.7 \\
\hline $\mathrm{FVC} \%$ & 11 & $524 / 512$ & 0.60 & $0.40,0.80$ & $<0.001$ & 56.2 \\
\hline FEV1\% & 10 & $393 / 388$ & 0.57 & $0.42,0.71$ & $<0.001$ & 14.1 \\
\hline TLC\% & 13 & $491 / 469$ & 0.27 & $0.15,0.40$ & $<0.001$ & 0 \\
\hline DLCO\% & 20 & $775 / 743$ & 0.38 & $0.28,0.48$ & $<0.001$ & 39.4 \\
\hline DLCO & 8 & $281 / 282$ & 1.19 & $1.01,1.37$ & $<0.001$ & 45.6 \\
\hline VC\% & 10 & $312 / 300$ & 0.35 & $0.19,0.51$ & $<0.001$ & 30.4 \\
\hline 6MWD & 11 & $424 / 404$ & 0.70 & $0.56,0.84$ & $<0.001$ & 23.3 \\
\hline SGRQ & 10 & $342 / 336$ & -0.51 & $-0.70,-0.22$ & $<0.001$ & 69.8 \\
\hline
\end{tabular}

TCM: traditional Chinese medicine; SMD: standard mean difference.

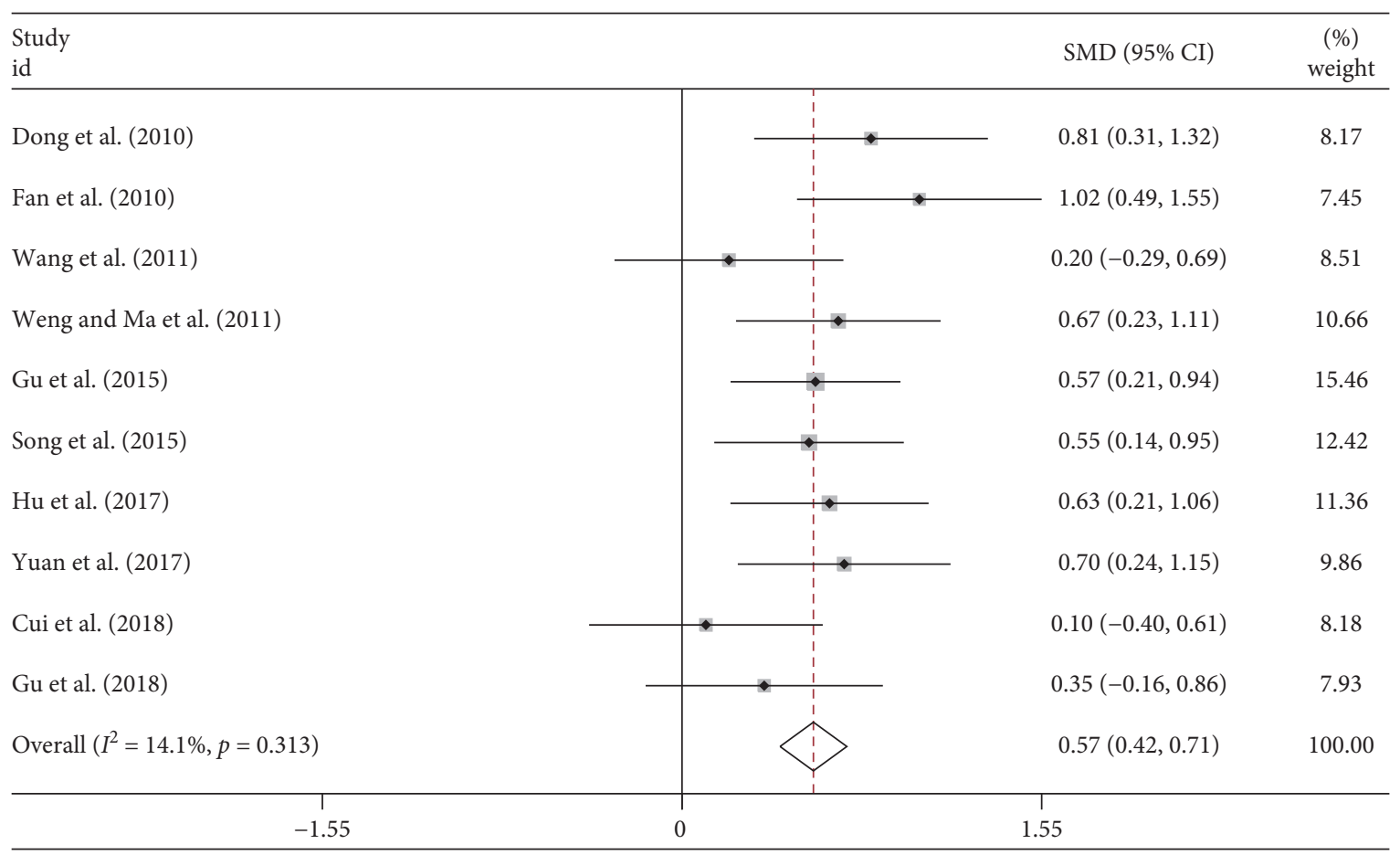

FIGURE 2: Forest plot of meta-analysis of FEV1\% difference between TCM and control groups. 


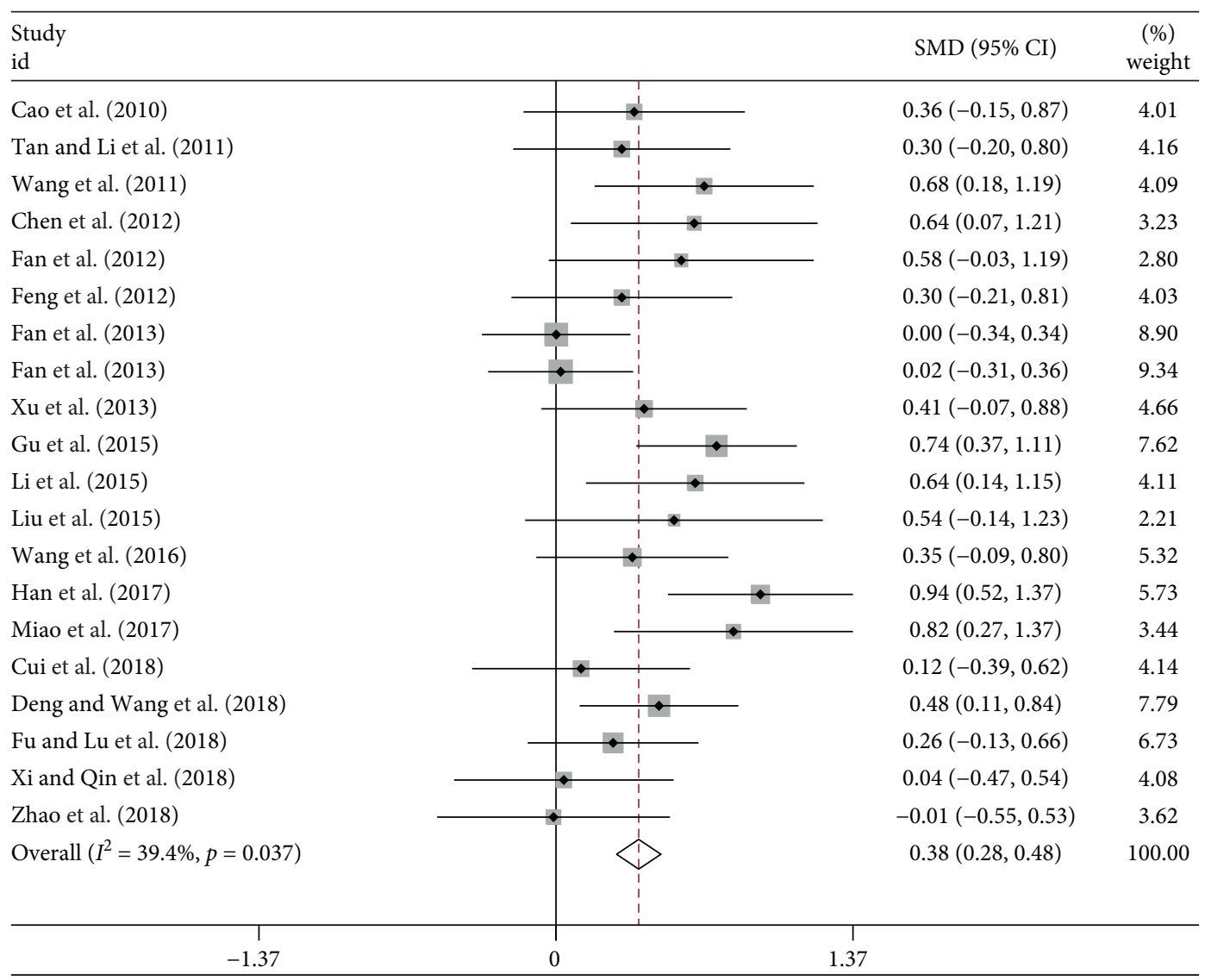

FIGURE 3: Forest plot of meta-analysis of DLCO $\%$ difference between TCM and control groups.

improvement of all measurements relating to lung function or life quality when compared with the control group in either the short-term subgroup or the long-term subgroup. In the TCM + WM vs. WM or TCM vs. placebo subgroup (Table 4), all measurements were significantly improved in TCM group. In the TCM vs. WM subgroup, similar results of each measurement were seen except $\mathrm{PO}_{2}$ and $\mathrm{VC} \%$ which showed no significant difference between both groups.

3.5. Safety. Only 14 studies reported the outcomes of adverse events (AEs), of which 6 observed no AEs and 8 reported various AEs, including stomach upset, nausea, dizziness, constipation, insomnia, slightly elevated blood glucose, and so on. Since the AE profiles varied among studies, only overall prevalence of AEs was compared between treatment and control groups. In the treatment group, 20 (3.4\%, 20/591) AEs were observed while 35 (7.2\%, 35/487) AEs were observed in the control group. In the two studies $[16,22]$, the TCM group had lower risk of AEs than the control group $(\mathrm{RR}=0.04$ (95\% CI: $0.00-0.63)$ and $\mathrm{RR}=0.11$ (95\% CI: 0.02-0.51), respectively). However, after pooled analysis of all studies using random-effect model, there was no significant difference of AEs between both groups $\left(\mathrm{RR}=0.66,95 \% \mathrm{CI}: 0.27-1.60, p=0.352, I^{2}=51.8 \%\right)$, indicating that TCM is well-tolerated.
3.6. Sensitivity Analysis and Publication Bias. We performed sensitivity analysis in each meta-analysis and found no single study could significantly affect the pooled results, indicating a high stability of our analysis. The funnel plots were symmetric and Egger's tests suggested no obvious publication bias.

\section{Discussions}

In recent decades, repetitive microinjuries to epithelium caused by the interaction of various environmental and genetic risk factors have been thought to play crucial role in the development of IPF [3]. These microinjuries cause aberrant communication between epithelium and fibroblast, the production of myofibroblasts, accumulation of extracellular matrix, and finally remodel lung interstitium [3]. With the development of the extraction and purification of herbs, TCM has been proved to be effective on IPF in animal models and exert some effect against the pathophysiology of IPF mentioned above [10]. These TCMs were found to inhibit the proliferation of fibroblast cells, downregulate the expression of matrix-related genes and induce the apoptosis of abnormal lung fibroblast [52, 53]. However, the clinical effect of TCM on IPF was controversial in spite of its wide usage in China. 


\begin{tabular}{|c|c|c|c|}
\hline \multicolumn{2}{|l|}{$\begin{array}{l}\text { Study } \\
\text { id }\end{array}$} & \multirow{2}{*}{$\begin{array}{l}\operatorname{SMD}(95 \% \mathrm{CI}) \\
0.83(0.32,1.33)\end{array}$} & \multirow{2}{*}{$\begin{array}{c}\begin{array}{c}(\%) \\
\text { weight }\end{array} \\
5.51\end{array}$} \\
\hline Dong et al. (2010) & - & & \\
\hline Tan and Li et al. (2011) & $\rightarrow$ & $0.26(-0.24,0.76)$ & 5.52 \\
\hline Weng and Ma et al. (2011) & & $0.81(0.36,1.26)$ & 5.76 \\
\hline Chen et al. (2012) & $\rightarrow 1$ & $0.65(0.08,1.22)$ & 5.20 \\
\hline Feng et al. (2012) & $\longrightarrow$ & $0.39(-0.12,0.90)$ & 5.47 \\
\hline Fan et al. (2013) & - & $-0.05(-0.39,0.29)$ & 6.19 \\
\hline Fan et al. (2013) & $\rightarrow$ & $0.19(-0.14,0.53)$ & 6.22 \\
\hline Xu et al. (2013) & & $0.66(0.18,1.14)$ & 5.60 \\
\hline Li et al. (2014) & & $0.63(0.03,1.22)$ & 5.07 \\
\hline Gu et al. (2015) & 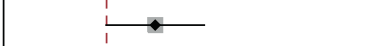 & $1.18(0.79,1.57)$ & 6.01 \\
\hline Liu et al. (2015) & & $0.54(-0.15,1.22)$ & 4.67 \\
\hline Sun et al. (2015) & 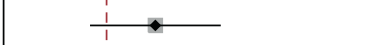 & $1.18(0.67,1.69)$ & 5.48 \\
\hline Hu et al. (2016) & & $0.76(0.31,1.21)$ & 5.73 \\
\hline Zhao and Wu et al. (2016) & $\longrightarrow$ & $1.92(1.39,2.45)$ & 5.38 \\
\hline Han et al. (2017) & $\longrightarrow$ & $1.43(0.98,1.89)$ & 5.72 \\
\hline Deng anf Wang et al. (2018) & - & $0.91(0.53,1.29)$ & 6.05 \\
\hline Wu et al. (2018) & $\longrightarrow$ & $2.05(1.42,2.68)$ & 4.93 \\
\hline Xi and Qin et al. (2018) & $\rightarrow$ & $0.31(-0.20,0.82)$ & 5.48 \\
\hline Overall $\left(I^{2}=81.7 \%, p=0.000\right)$ & & $0.80(0.54,1.06)$ & 100.00 \\
\hline Note: weights are from random effects analysis & & & \\
\hline $\begin{array}{r}1 \\
-2.68\end{array}$ & $\begin{array}{c}1 \\
2.68\end{array}$ & & \\
\hline
\end{tabular}

FIGURE 4: Forest plot of meta-analysis of $\mathrm{PO}_{2}$ difference between TCM and control groups.

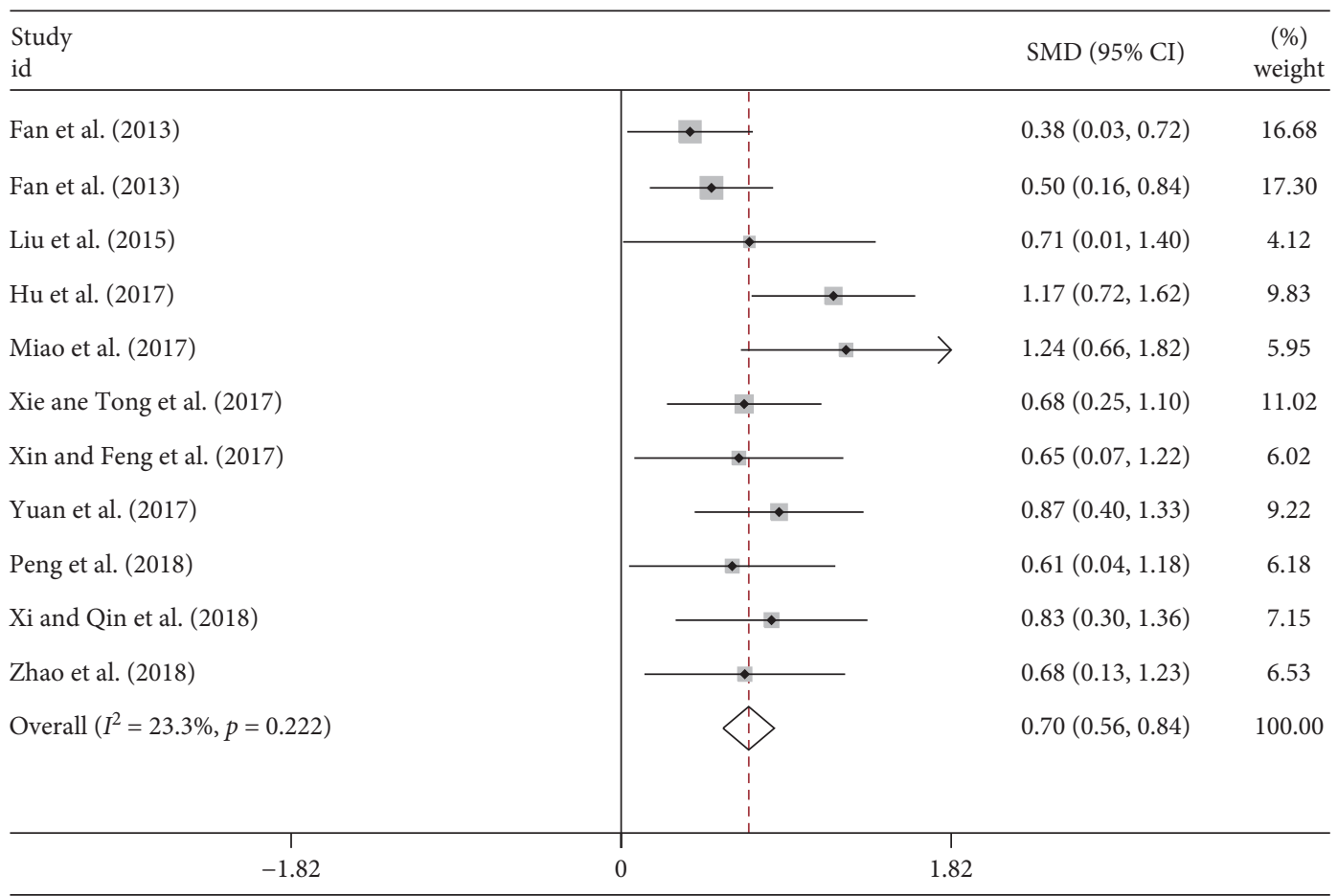

Figure 5: Forest plot of meta-analysis of 6MWD difference between TCM and control groups. 


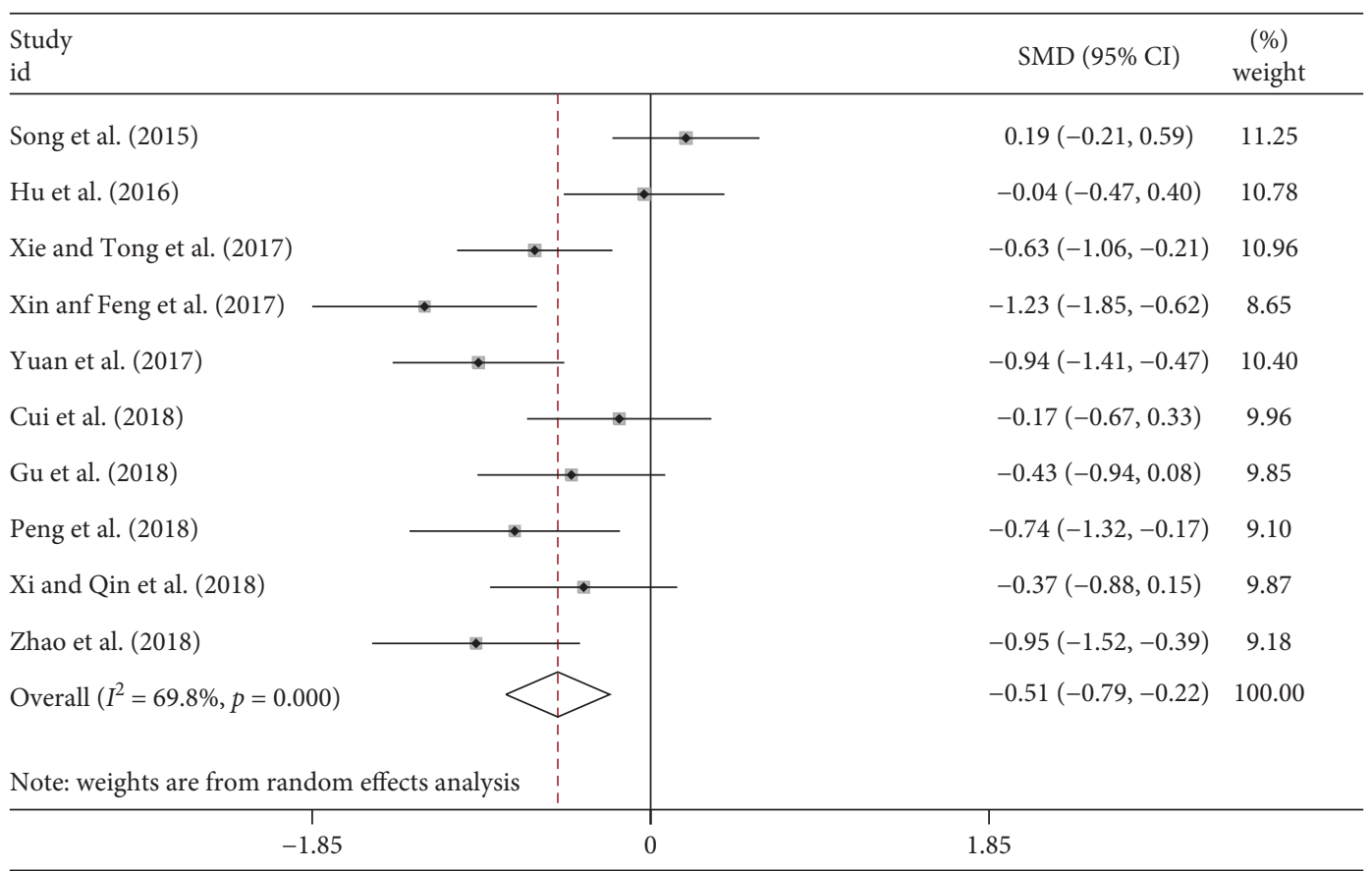

FIGURE 6: Forest plot of meta-analysis of SGRQ scores difference between TCM and control groups.

TABLE 3: Subgroup analysis of the efficacy of TCM on idiopathic pulmonary fibrosis according to study duration*.

\begin{tabular}{|c|c|c|c|c|c|c|}
\hline \multirow{2}{*}{ Measurements } & \multicolumn{3}{|c|}{3 months } & \multicolumn{3}{|c|}{6 months or more } \\
\hline & SMD $(95 \% \mathrm{CI})$ & $p$ & $I^{2}(\%)$ & SMD $(95 \%$ CI $)$ & $p$ & $I^{2}(\%)$ \\
\hline $\mathrm{PO}_{2}$ & $0.82(0.50,1.14)$ & $<0.001$ & 84.8 & $0.77(0.41,1.14)$ & $<0.001$ & 55.0 \\
\hline FEV1/FVC & $1.31(1.04,1.59)$ & $<0.001$ & 45.8 & $0.50(0.22,0.78)$ & $<0.001$ & 15.1 \\
\hline $\mathrm{FVC} \%$ & $0.50(0.15,0.86)$ & 0.005 & 71.3 & $0.75(0.58,0.92)$ & $<0.001$ & 0 \\
\hline FEV1\% & $0.55(0.32,0.79)$ & $<0.001$ & 33.2 & $0.58(0.39,0.76)$ & $<0.001$ & 15.9 \\
\hline TLC\% & $0.25(0.12,0.38)$ & $<0.001$ & 0 & - & - & - \\
\hline DLCO\% & $0.33(0.22,0.45)$ & $<0.001$ & 44.8 & $0.56(0.34,0.78)$ & $<0.001$ & 0 \\
\hline DLCO & $1.23(1.04,1.42)$ & $<0.001$ & 48.8 & - & - & - \\
\hline $\mathrm{VC} \%$ & $0.34(0.17,0.50)$ & $<0.001$ & 36.9 & - & - & - \\
\hline 6MWD & $0.65(0.49,0.81)$ & $<0.001$ & 17.0 & $0.92(0.60,1.23)$ & $<0.001$ & 16.3 \\
\hline SGRQ & $-0.44(-0.74,-0.13)$ & 0.005 & 69.7 & - & - & - \\
\hline
\end{tabular}

${ }^{*}$ Only the subgroups involving at least 3 studies were analyzed; TCM: traditional Chinese medicine.

TABLE 4: Subgroup analysis of the efficacy of TCM on idiopathic pulmonary fibrosis according to comparison models*.

\begin{tabular}{|c|c|c|c|c|c|c|}
\hline \multirow{2}{*}{ Measurements } & \multicolumn{3}{|c|}{ TCM vs. WM } & \multicolumn{3}{|c|}{ TCM + WM vs. WM or TCM vs. Placebo } \\
\hline & SMD $(95 \% \mathrm{CI})$ & $p$ & $I^{2}(\%)$ & SMD $(95 \% \mathrm{CI})$ & $p$ & $I^{2}(\%)$ \\
\hline $\mathrm{PO}_{2}$ & $0.35(-0.03,0.73)$ & 0.072 & 68.7 & $0.93(0.66,1.20)$ & $<0.001$ & 75.9 \\
\hline FEV1/FVC & - & - & - & $0.98(0.52,1.44)$ & $<0.001$ & 78.7 \\
\hline $\mathrm{FVC} \%$ & $0.52(0.23,0.82)$ & 0.001 & 38.5 & $0.62(0.38,0.87)$ & $<0.001$ & 62.8 \\
\hline FEV1\% & $0.67(0.19,1.16)$ & 0.007 & 63.6 & $0.53(0.37,0.70)$ & $<0.001$ & 0 \\
\hline TLC\% & $0.19(0.02,0.36)$ & 0.028 & 0 & $0.37(0.18,0.56)$ & $<0.001$ & 24.4 \\
\hline DLCO $\%$ & $0.33(0.10,0.57)$ & 0.006 & 53.5 & $0.46(0.33,0.60)$ & $<0.001$ & 16.8 \\
\hline DLCO & $1.19(1.01,1.37)$ & $<0.001$ & 45.6 & - & - & - \\
\hline $\mathrm{VC} \%$ & $0.39(0.00,0.79)$ & 0.050 & 50.6 & $0.33(0.14,0.53)$ & 0.001 & 26.5 \\
\hline 6MWD & $0.58(0.39,0.78)$ & $<0.001$ & 40.0 & $0.83(0.63,1.04)$ & $<0.001$ & 0 \\
\hline SGRQ & - & - & - & $-0.38(-0.65,-0.10)$ & 0.008 & 63.5 \\
\hline
\end{tabular}

${ }^{*}$ Only the subgroups involving at least 3 studies were analyzed; TCM: traditional Chinese medicine; WM: western medicine. 
In present study, we systematically reviewed and pooled the results of 40 studies comprising 3194 individuals to comprehensively evaluate the efficacy of TCM on the improvement of lung function and life quality of IPF. We found that TCM had significant impact on the change of lung function, exercise capacity, and life quality before and after treatment. After pooling analysis, there was significant difference between the change of $\mathrm{PO}_{2}, \mathrm{FEV} 1 / \mathrm{FVC}, \mathrm{FVC} \%$, FEV1\%, TLC\%, DLCO\% or DLCO, and VC\% in the TCM and control groups. Similar results were observed when TCM was used alone or in combination with western medicines, indicating TCM may help improve lung function or slow the decline of lung function of IPF. On the other side, there was significant improvement of 6MWD and decrease of SGRQ scores in the TCM treatment group compared with the control group, suggesting that TCM can improve the exercise capacity and quality of life of IPF patients. In addition, we found similar risk of adverse events between TCM and control groups, indicating that TCM is safe for IPF patients. There was no obvious heterogeneity and publication bias in most of the pooling analyses. Sensitivity analysis suggested that these pooled results were stable.

However, there were some limitations in the present study. Firstly, the quality of the majority of included studies was poor because the blindness was unclear and dropouts were not mentioned. More well-designed, double-blinded, multicentered RCTs in high quality are needed. Secondly, we pooled all TCMs together in the present study instead of analyzing one single TCM compounds due to limited number of studies related to each compound. TCM compounds are complicated and may have various effects on IPF. The present study may over- or underestimate the effect of some certain compounds. Meta-analysis aiming to precisely evaluate the efficacy of one single TCM compound on IPF is necessary. Thirdly, only a small proportion of studies (35\%) reported the outcomes of adverse events and the safety of TCM needs to be confirmed by more studies. Fourthly, moderate-to-high heterogeneity existed in most of the analyses and the interpretation of our results needs caution. Finally, the quality of life was only assessed using SGRQ scores. The other measurements that may be more appropriate, including CAT, SF-36, and ATAQ-IPF, were not analyzed due to insufficient data. In the future, more studies using these tools may be needed.

\section{Conclusions}

In conclusion, we performed a meta-analysis involving 40 RCTs, which may have the largest sample size as far as we know, and found that TCM treatment may help improve the lung function, exercise capacity, and quality of life in IPF patients and the treatment is safe. However, more randomizes controlled clinical trials are needed for in the future.

\section{Data Availability}

The data used to support the findings of this study are included within the article.

\section{Conflicts of Interest}

All authors declare no conflicts of interest.

\section{Authors' Contributions}

Kun Ji and Jianling Ma contributed equally to this work. KJ and JM analyzed and interpreted the data, drafted the manuscript. KL and JM performed literature search and data extraction. LW, NL, and SD interpreted the data and revised the manuscript critically. LS designed the study, revised critically, and made final approval of the manuscript. All authors read and approved the final manuscript.

\section{Acknowledgments}

This work was supported by National Pilot Project of Clinical Collaboration of Traditional Chinses Medicine and Western Medicine on Major and Difficult Diseases (Pulmonary Fibrosis) (no. 2018), National Natural Science Foundation of China (no. 81373588), and Dongfang Hospital 1166 Talent Training Project (no. 2018).

\section{References}

[1] J. Hutchinson, A. Fogarty, R. Hubbard, and T. McKeever, "Global incidence and mortality of idiopathic pulmonary fibrosis: a systematic review," European Respiratory Journal, vol. 46, no. 3, pp. 795-806, 2015.

[2] R. B. Hopkins, N. Burke, C. Fell, G. Dion, and M. Kolb, "Epidemiology and survival of idiopathic pulmonary fibrosis from national data in Canada," European Respiratory Journal, vol. 48, no. 1, pp. 187-195, 2016.

[3] L. Richeldi, H. R. Collard, and M. G. Jones, "Idiopathic pulmonary fibrosis," The Lancet, vol. 389, no. 10082, pp. 1941-1952, 2017.

[4] B. Ley, H. R. Collard, and T. E. King, "Clinical course and prediction of survival in idiopathic pulmonary fibrosis," American Journal of Respiratory and Critical Care Medicine, vol. 183, no. 4, pp. 431-440, 2011.

[5] K. J. Anstrom, T. E. King, J. A. Lasky, F. J. Martinez, and Idiopathic Pulmonary Fibrosis Clinical Research Network, "Prednisone, azathioprine, and $\mathrm{N}$-acetylcysteine for pulmonary fibrosis," New England Journal of Medicine, vol. 366, no. 21, pp. 1968-1977, 2012.

[6] I. Noth, K. J. Anstrom, S. B. Calvert et al., "A placebo-controlled randomized trial of warfarin in idiopathic pulmonary fibrosis," American Journal of Respiratory and Critical Care Medicine, vol. 186, no. 1, pp. 88-95, 2012.

[7] T. E. King, W. Z. Bradford, S. Castro-Bernerdini et al., "A phase 3 trial of pirfenidone in patients with idiopathic pulmonary fibrosis," New England Journal of Medicine, vol. 370, no. 2, pp. 2083-2092, 2014.

[8] L. Richeldi, R. M. du Bois, G. Raghu et al., "Efficacy and safety of nintedanib in idiopathic pulmonary fibrosis," New England Journal of Medicine, vol. 370, no. 22, pp. 2071-2082, 2014.

[9] K. D. Kistler, L. Nalysnyk, P. Rotella, and D. Esser, "Lung transplantation in idiopathic pulmonary fibrosis: a systematic review of the literature," BMC Pulmonary Medicine, vol. 14, no. 1, p. 139, 2014.

[10] H. B. Huang, X. N. Peng, and C. W. Zhong, "Idiopathic pulmonary fibrosis: the current status of its epidemiology, 
diagnosis, and treatment in China," Intractable \& Rare Diseases Research, vol. 2, no. 3, pp. 88-93, 2013.

[11] A. R. Jadad, R. A. Moore, D. Carroll et al., "Assessing the quality of reports of randomized clinical trials: is blinding necessary?" Controlled Clinical Trials, vol. 17, no. 1, pp. 1-12, 1996.

[12] Z. H. Cao, D. Han, B. H. Zhang, and Z. X. Yang, "Clinical observation of Tongluo Huaxian Granules in the treatment of 60 cases with idiopathic pulmonary fibrosis," Heilongjiang Journal of Traditional Chinese Medicine, vol. 39, no. 2, pp. 22-23, 2010.

[13] H. Dong, "Clinical observation of Kangxian Shufei Granules in the treatment of idiopathic pulmonary fibrosis," Chinese Journal of Information on Traditional Chinese Medicine, vol. 17, no. 3, pp. 60-61, 2010.

[14] D. B. Fan, X. P. Qin, H. H. Bai et al., "Clinical observation of Kechuanting Granules in the treatment of 32 cases with idiopathic pulmonary fibrosis," Journal of Emergency Traditional Chinese Medicine, vol. 19, no. 6, p. 921, 2010.

[15] J. Tan and H. J. Li, "The influence of Shuizhi Tongluo Capsule on the lung function of patients with idiopathic pulmonary fibrosis," Jounal of Sichuan Traditional Chinese Medicine, vol. 29, no. 3, pp. 83-84, 2011.

[16] H. Wang, Y. H. Guo, and Y. Zhu, "Clinical observation of Yangyin Yiqi Misture in treating idiopathic pulmonary fibrosis with qi and yin deficiency," China Journal of Traditional Chinese Medicine and Pharmacy, vol. 26, no. 3, pp. 620-622, 2011.

[17] H. Weng and D. F. Ma, "Clinical observation of Qingjin Decoction in treating idiopathic pulmonary fibrosis," Journal of Gansu College of Traditional Chinese Medicine, vol. 24, no. 1, pp. 40-41, 2011.

[18] P. Chen, G. L. Xu, and Q. Wang, "Study on curative effect of Huaxian Pogu Formula on patients with idiopathic pulmonary fibrosis," Liaoning Journal of Traditional Chinese Medicine, vol. 39, no. 11, pp. 2194-2195, 2012.

[19] M. R. Fan, Q. Miao, H. L. Luo, S. C. Wang, and Y. P. Zhang, "Clinical observation of Feixiantong Decoction on idiopathic pulmonary fibrosis with qiyinliangxu and feiluobizu syndrome," Journal of Emergency Traditional Chinese Medicine, vol. 21, no. 9, pp. 1377-1379, 2012.

[20] M. Feng, Y. X. Zhang, C. G. Liu, and C. X. Wang, "Clinical observation of Feixiankang Granules in treating idiopathic pulmonary fibrosis," Guide of Chinese Medicine, vol. 10, no. 10, pp. 631-632, 2012.

[21] L. P. Gan, Z. Q. Cao, and L. Wang, "Therapeutic effect of Huaxian Decoction in the treatment of 28 cases with idiopathic pulmonary fibrosis," Journal of New Chinese Medicine, vol. 44, no. 5, pp. 23-24, 2012.

[22] M. R. Fan, Y. P. Zhang, Q. Miao et al., "Clinical trial of Feitong oral liquid in treating idiopathic pulmonary fibrosis," Traditional Chinese Drug Research \& Clinical Pharmacology, vol. 24, no. 3, pp. 317-322, 2013.

[23] Y. L. Xu, K. M. Zhao, X. Zhen et al., "Invigorating lung and kidney and activating blood circulation decoction combined with western med in the treatment of idiopathic pulmonary interstitial fibrosis (IPF) randomized parallel controlled study," Journal of Practice in Traditional Chinese Internal Medicine, vol. 27, no. 20, pp. 43-46, 2013.

[24] P. Chen, "Curative effect of Danhong injection in the treatment of idiopathic pulmonary fibrosis," Clinical Pulmonary Medicine, vol. 19, no. 2, pp. 319-321, 2014.

[25] Y. Y. Li, Y. P. Zhang, S. C. Wang et al., "YiQiTongLuo method in treating idiopathic pulmonary fibrosis: a clinical trial,"
Lishizhen Medicine and Materia Medica Research, vol. 25, no. 3, pp. 630-632, 2014.

[26] X. X. Yan, F. Yuan, and F. J. Zheng, “Assessment of Buyang Huanwu Decoction combining $\mathrm{N}$-acetylcysteine in treating idiopathic pulmonary fibrosis," Internal Medicine, vol. 10, no. 4, pp. 468-470, 2015.

[27] Z. H. Li, R. Dong, F. R. Xin et al., "Observation of curative effect of Yangyin Yifei Tongluo Wan on patients with idiopathic pulmonary fibrosis," Journal of Liaoning University Traditional Chinese Medicine, vol. 17, no. 11, pp. 163-165, 2015.

[28] M. Liu, Q. L. Zhao, G. Y. Liu et al., "Clinical observation of Xuefu Zhuyu Capsule combined with $\mathrm{N}$-acetylcysteine in the treatment of idiopathic pulmonary fibrosis," China Journal of Traditional Chinese Medicine and Pharmacy, vol. 30, no. 2, pp. 473-476, 2015.

[29] Y. Y. Song, "The clinical research on quality of life improvement of patients with idiopathic pulmonary fibrosis treated with Xuefu Zhuyu Decoction," Journal of Emergency in Traditional Chinese Medicine, vol. 24, no. 9, pp. 1627-1629, 2015.

[30] Y. Sun, Y. Q. Li, and X. D. Lv, "Clinical effect of Danhong injection combined with edaravone in patients with idiopathic pulmonary fibrosis and influence on TNF- $\alpha$ and TGF- $\beta 1$ in serum," Chinese Journal of Biochemical Pharmaceutics, vol. 35, no. 10, pp. 38-40, 2015.

[31] P. Yan, "Analysis of the therapeutic effect of Danhong Injection on idiopathic pulmonary fibrosis," China Modern Doctor, vol. 53, no. 22, pp. 123-125, 2015.

[32] W. L. Hu, S. S. Zhao, M. L. Tu, and Q. Wang, "Effect of supplementing qi and removing blood stasis and dredging collateral decoction on $\mathrm{T}$ lymphocyte subsets of patients with idiopathic pulmonary fibrosis," Information Traditional Chinese Medicine, vol. 33, no. 6, pp. 62-66, 2016.

[33] Q. J. Wang, P. Li, and X. Liu, "Randomized controlled trial: therapeutic observation of Wenyang Huayu Decoction in the treatment of 80 cases with idiopathic pulmonary interstitial fibrosis," China Continent Medical Education, vol. 8, no. 3, pp. 193-196, 2016.

[34] L. Zhao and Z. Wu, "Effects of combination of acetylcysteine and Danhong Injection on pulmonary function and serum levels of TNF- $\alpha$ and TGF- $\beta 1$ in patients with IPF," Journal of Hainan Medical University, vol. 22, no. 23, pp. 2811-2813, 2016.

[35] Z. G. Han, W. J. Peng, and X. F. Chu, "The curative effect of Pingfeng Shengmai Powder combined with prednisone in treating idiopathic pulmonary fibrosis and the influence on T cell subset," Modern Journal of Integrated Traditional Chinese and Western Medicine, vol. 26, no. 24, pp. 2714-2716, 2017.

[36] Y. Q. Hu, “The effect of pingfeng shengmai powder combing prednisone in the treatment of idiopathic pulmonary fibrosis and on the expression of T cell subset," Shanxi Journal of Traditional Chinese Medicine, vol. 38, no. 7, pp. 889-890, 2017.

[37] W. J. Li, M. Liu, and Y. X. Wang, "Effects of Peiyuan Huoxue decoction combined with prednisone on idiopathic pulmonary fibrosis and on the expression of coagulation function and serum SP-A, MMP-9 and VCAM-1," Modern Journal of Integrated Traditional Chinese and Western Medicine, vol. 26, no. 34, pp. 3786-3789, 2017.

[38] Q. Miao, X. D. Cong, M. R. Fan et al., "Therapeutic observation of Bushen Tongluo Decoction in the treatment of 28 cases with idiopathic pulmonary fibrosis," Lishizhen Medicine 
and Materia Medica Research, vol. 28, no. 2, pp. 395-397, 2017.

[39] L. L. Wang, "Therapeutic effect of prednisone combined with Xuefu Zhuyu Decoction in the treatment of idiopathic pulmonary fibrosis," Journal of Practice in Traditional Chinese Medicine, vol. 33, no. 10, pp. 1147-1148, 2017.

[40] Y. G. Xie and Z. Tong, "Clinical observation of Yangyin Yiqi mixture for IPF with qi-yin deficiency syndrome," Journal of New Chinese Medicine, vol. 49, no. 5, pp. 38-40, 2017.

[41] D. Y. Xin and J. S. Feng, “Tonifying qi, softening hardness and eliminating abdominal mass to improve life of patients of idiopathic pulmonary fibrosis with qi deficiency and blood stasis: a clinical study," World Chinese Medicine, vol. 11, no. 12, pp. 2714-2716, 2016.

[42] M. Yuan, S. Y. Yuan, and L. Y. Zhu, "Clinical research on the effect of Bufei Yishen Huoxue misture in treating idiopathic pulmonary fibrosis," Guide to Traditional Chinese Medicine and Pharmacy, vol. 23, no. 11, pp. 98-100, 2017.

[43] L. F. Cui, B. L. Zhang, L. Y. Wang et al., "Clinical observation of the quality of life in the patients of idiopathic pulmonary fibrosis in the intervention of herbal med," World Journal of Integrated Traditional Western Medicine, vol. 13, no. 1, pp. 88-91, 2018.

[44] F. Deng and Y. Wang, "The therapeutic effect of Huangqi Taohong Decoction combined with prednisone in treating idiopathic pulmonary fibrosis and the influence on the expression of IGF-1 and IGFBP-4 in bronchoalveolar lavage fluid," Modern Journal of Integrated Traditional Chinese Western Medicine, vol. 27, no. 18, pp. 2034-2036, 2018.

[45] J. Feng and G. Sun, "Clinical observation of Yifei Tongluo Recipe in the treatment of idiopathic pulmonary fibrosis with qi-yin deficiency type," Hebei Journal of Traditional Chinese Medicine, vol. 40, no. 12, pp. 1803-1806, 2018.

[46] L. Fu and L. Luo, "Clinical trial of Loubei Lengshu Decoction in treating idiopathic pulmonary fibrosis and the influence on serum level of TGF- $\beta 1$," Ming Yi, vol. 4, pp. 54-55, 2018.

[47] C. H. Gu, "Effect of Qishufeixian decoction combined with pirfenidone on the treatment of idiopathic pulmonary fibrosis," Journal of Sichuan Traditional Chinese Medicine, vol. 36, no. 9, pp. 75-77, 2018.

[48] Y. F. Peng, Y. Y. Yue, Y. W. Zhang, W. Ai, and Y. Q. Zhao, "Clinical study on the treatment of idiopathic pulmonary fibrosis treated with Qigui Recipe combined with prednisone," World Chinese Medicine, vol. 13, no. 8, pp. 1908-1912, 2018.

[49] B. P. Wu, "Efficacy and safety of Danhong injection on the treatment of idiopathic pulmonary fibrosis," Chinese Journal of Clinical Rational Drug Use, vol. 11, no. 2, pp. 60-61, 2018.

[50] N. Xi and X. J. Qin, "Clinical observation of huaxian tongluo decoction combined with acetylcysteine in the treatment of idiopathic pulmonary fibrosis," Journal of Liaoning University Traditional Chinese Medicine, vol. 20, no. 12, pp. 201-204, 2018.

[51] G. J. Zhao, H. B. Hu, P. Han, and X. C. Lu, "Clinical study of idiopathic pulmonary fibrosis treated with Fuzheng Huaxian formula," Shandong Journal of Traditional Chinese Medicine, vol. 37, no. 1, pp. 22-25, 2018.

[52] Y.-x. Ren, R. Zhou, W. Tang et al., “(5R)-5-hydroxytriptolide (LLDT-8) protects against bleomycin-induced lung fibrosis in mice," Acta Pharmacologica Sinica, vol. 28, no. 4, pp. 518-525, 2007.

[53] Y. Jiang, W. Li, and M. Chen, "Effect of ligustrazine on CTGF expression and collagen deposition in rats with pulmonary fibrosis," Chinese Journal of General Practitioners, vol. 6, no. 12, pp. 1215-1216, 2008. 